\title{
Human Anterior Cingulate Cortex Neurons Encode Cognitive and Emotional Demands
}

\author{
Karen D. Davis, ${ }^{1,2}$ Keri S. Taylor, ${ }^{1}$ William D. Hutchison, ${ }^{1,2,3}$ Jonathan 0. Dostrovsky, ${ }^{1,2,3}$ Mary P. McAndrews, ${ }^{1,4}$ \\ Erich 0. Richter, ${ }^{1,2}$ and Andres M. Lozano ${ }^{1,2}$ \\ ${ }^{1}$ Toronto Western Research Institute and Departments of ${ }^{2}$ Surgery, ${ }^{3}$ Physiology, and ${ }^{4}$ Psychology, University of Toronto, Toronto, Ontario, \\ Canada M5T 2S8
}

\begin{abstract}
The cortical mechanisms and substrates of cognitive and emotional demands are poorly understood. Lesion studies and functional imaging implicate the anterior cingulate cortex (ACC). The caudal ACC (cACC) has been implicated in cognitive processes such as attention, salience, interference, and response competition, mostly on the basis of neuroimaging results. To test the hypothesis that individual cACC neurons subserve these functions, we monitored neuronal activity from single cells in the cACC while subjects were engaged in a mental arithmetic task, the cognitively demanding counting Stroop task, and/or the emotional Stroop interference task. We now report the first direct measures of single neurons in humans identifying a population of cACC neurons that respond differentially or in a graded manner to cognitively demanding high- and low-conflict Stroop tasks, including those with emotional valence. These data indicate that cACC neurons may be acting as salience detectors when faced with conflict and difficult or emotional stimuli, consistent with neuroimaging results of cACC responses to abrupt sensory, novel, task-relevant, or painful stimuli.
\end{abstract}

Key words: cognition; attention; emotion; cingulate; OCD; Stroop; salience

\section{Introduction}

The anterior cingulate cortex (ACC) has been implicated in cognitive, affective, and emotional processes. Anatomical, neuroimaging, and lesion data have led to the concept of two major ACC subdivisions: a rostral affect division and a caudal cognitive division (Bush et al., 2000). A fundamental question is whether particular regions of the ACC contribute to specific or general cognitive functions. The caudal ACC (cACC) has been implicated in cognitive processes such as attention, salience, interference, and response competition, mostly on the basis of neuroimaging results (Bush et al., 2000; Casey et al., 2000; Braver et al., 2001; Ruff et al., 2001; Downar et al., 2002; Botvinick et al., 2004). The details of cingulate functionality not only shape our basic understanding of limbic function but also impact clinical decisions. For example, surgical midcaudal cingulotomy alleviates symptoms in some but not all patients with obsessive-compulsive disorder (OCD) or chronic pain (Pillay and Hassenbusch, 1992; Richter et al., 2004), and subgenual cingulate stimulation has had initial clinical success in the treatment of depression (Mayberg et al., 2005); therefore, a better understanding of ACC neuronal function will assist in developing more effective treatments for neurological and psychiatric disorders.

Scant information is available about the behavior of single

Received June 7, 2005; revised July 29, 2005; accepted July 31, 2005.

This work was supported by the Ontario Mental Health Foundation. K.D.D. is a Canada Research Chair in Brain and Behaviour.

Correspondence should be addressed to Dr. Karen D. Davis, Toronto Western Hospital, University Health Network, 399 Bathurst Street, Room MP14-306, Toronto, 0ntario, Canada M5T 2S8. E-mail: kdavis@uhnres.utoronto.ca. DOI:10.1523/JNEUROSCI.2315-05.2005

Copyright $\odot 2005$ Society for Neuroscience $\quad$ 0270-6474/05/258402-05\$15.00/0 neurons in the human ACC because of the obvious limitation imposed by invasive electrophysiological cell recording techniques. The three studies to date report dorsal ACC rewardrelated responses (Williams et al., 2004), cACC pain-related responses (Hutchison et al., 1999), and simple attention-related responses (mental arithmetic) (Davis et al., 2000); however, very few of the tested cACC neurons responded to simple tasks. We therefore hypothesized that human cACC neurons play a role in more complex or demanding functions. We tested whether cACC neurons were specifically responsive to complex attentiondemanding cognitive tasks, and we tested the impact of emotional valence. We used the counting Stroop (CS) task, the emotional Stroop (ES) task, and an OCD-specific variant of the ES task, because these have been used in imaging studies to map the involved regions of the ACC (Bush et al., 1998; Whalen et al., 1998).

\section{Materials and Methods}

Electrophysiological recordings were made with tungsten microelectrodes in 16 electrode trajectories ( 9 right, 7 left) through the cACC in nine patients undergoing bilateral stereotactic cingulotomy for treatment of medically intractable, severe OCD. Surgical and recording procedures have been described previously (Davis et al., 2000; Richter et al., 2004). The surgical target was near the center of the cingulate gyrus as visualized by coronal magnetic resonance imaging (MRI) slice taken 2-4 $\mathrm{cm}$ posterior to the anterior tip of the frontal horn, and the recording sites have been estimated to be within the region $x=3-5 \mathrm{~mm}, y=3-13$ $\mathrm{mm}$, and $z=26-36 \mathrm{~mm}$ in Talairach space (Hutchison et al., 1999; Davis et al., 2000; Richter et al., 2004). Recording sites were confirmed to be within the cACC on the basis of preoperative MRI-based stereotactic coordinates and microelectrode microdrive recording depth readouts.

Neuronal activity was then recorded while patients were engaged in 


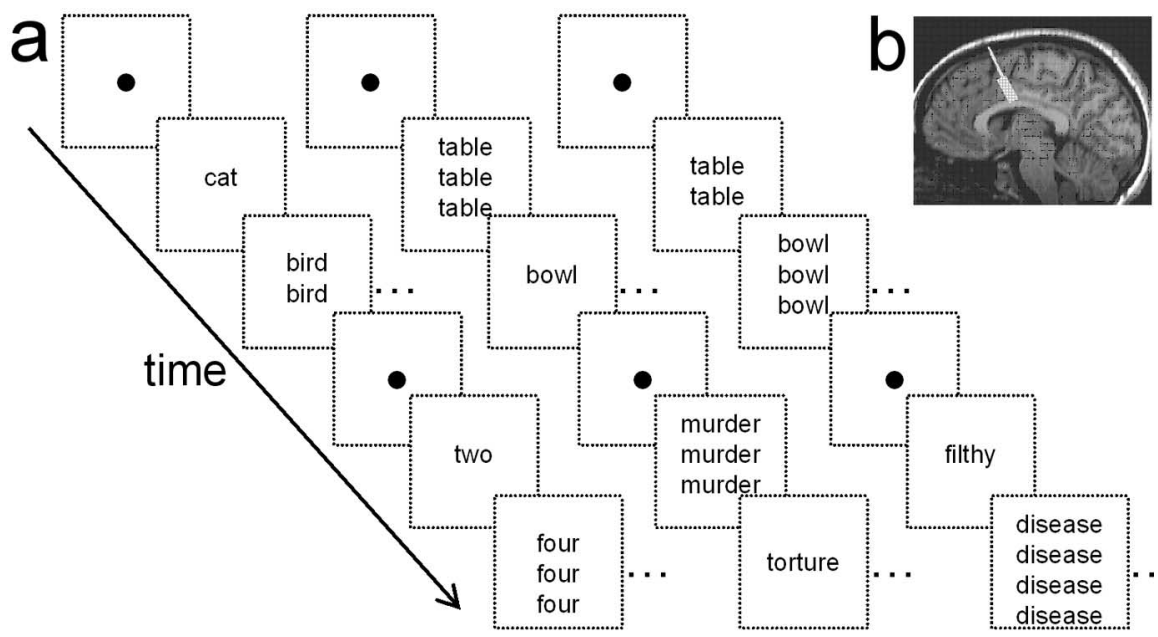

Figure 1. Stroop tasks and recording sites. $\boldsymbol{a}$, Schematic diagram depicts the counting, general-emotional, and OCD-emotional and Stroop tasks. Each diagonal sequence of boxes represents an example of the series of low-conflict words (animals or household items) and high-conflict words (numbers or emotional) displayed to the subject. Conflict-interference is introduced in the CS task through a discordance between the word content and the number of instances of the word on the screen. In the ES task, the conflict-interference arises from the emotional valence of the word. $\boldsymbol{b}$, Representative example showing the electrode trajectory (white line) and area of recorded cells (rectangle) in one subject reconstructed from MRI and cell microdrive recording depths.

one or more (determined by surgical time restrictions) attentiondemanding cognitive tasks. During the recording sessions, practice trials were used with verbalization to ensure adequate performance. The patient was then instructed to perform the tasks silently to avoid microelectronic noise created by vocalization.

Each attention-demanding task was repeated several times; however, tasks were not repeated more than three times per cell to avoid potential habituation effects and to minimize the overall surgical time. The mental arithmetic task was counting backward by 3 s from 100. The Stroop tasks required the patient to count the number of times (one to four) a word appeared in a vertical list displayed on a laptop screen (Fig. 1). Each word list was displayed for 1-2 s within 10-30 s blocks of either low conflict (neutral) or high conflict (interference) interleaved with control blocks when the patient observed a fixation point. The high-conflict words introduced cognitive interference and difficulty. In the CS task, the highconflict words were number words ("one," "two," "three," and "four") discordant with the number of instances shown on the screen, and the low-conflict neutral words were animal names (e.g., dog, cat, bird). In the ES task, the low-conflict words were household items (e.g., pan, table, curtain). In the general ES task, the high-conflict words were emotionally charged (e.g., murder, pain, torture); in the OCD ES task, the highconflict words had particular emotional valence in OCD (e.g., filthy, disease, sweat) (Whalen et al., 1998). The Stroop tasks were administered to a subset $(n=7)$ of the participants as part of a neuropsychological evaluation at least 1 week before the recording sessions. Tasks were performed at $98 \%$ accuracy in the high-conflict CS task and at 100\% accuracy in the low- and other high-conflict (ES) tasks. Reaction times for the high-conflict CS $[908 \pm 63 \mathrm{~ms}$ (mean $\pm \mathrm{SE})]$, general ES $(870 \pm 81 \mathrm{~ms})$, and OCD ES $(834 \pm 77 \mathrm{~ms})$ tasks were not significantly different from each other ( $p>0.1$; Wilcoxon signed rank test); however, reaction times for these three high-conflict conditions were significantly slower than for the low-conflict (neutral word) condition $(756 \pm 54 \mathrm{~ms})(p<0.03$; Wilcoxon signed rank test), which confirms the typical conflict cost seen in these types of tasks.

During the recording sessions, a statistical comparison ( $t$ test) was made between the weighted average of the raw firing rate during the task blocks and control blocks for each task in each cell. A single-factor ANOVA was then performed separately for each cell (or cell grouping) on the incidence of cells, cell firing rates, and percentage change in firing rates for the task-responsive cells.

\section{Results}

A total of 50 single cells in the cACC were recorded while patients were engaged in one or more tasks. These tasks altered the baseline (or fixation block) neuronal activity in $84 \%$ of the cells (Table 1 ). Figure 2 shows examples of excitatory and inhibitory task-related responses. Cellular firing rates were modulated for $68 \%$ of the 25 cells tested in the mental arithmetic task, $59 \%$ of the 32 cells tested in the lowconflict CS or ES task, $30 \%$ of the 20 cells testing during the high-conflict CS task, $54 \%$ of the 24 cells during the highconflict ES task, and $44 \%$ of the 25 cells tested during the high-conflict OCD ES task (Tables 2, 3). One-half of these multitask-tested cells responded (inhibitory or excitatory) to more than one task. The overall (group averaged) magnitude of task-related changes in firing rate did not differ across tasks for either the excitatory responses $(p=0.11)$ or the inhibitory responses ( $p=0.50)$; however, overall the task-related inhibitory responses $(40 \pm 5 \%$ decreased rate; $n=27)$ were relatively modest compared with the excitatory responses across tasks ( $141 \pm 7 \%$ increased rate; $n=45)$. This may be attributable in part to relatively low baseline activity levels (inhibited cells, $10.8 \pm 31.3 \mathrm{~Hz}$; excited cells, $5.6 \pm 3.4 \mathrm{~Hz}$ ).

Cells were classified on the basis of whether they showed statistically significant responses (inhibitory or excitatory) during the task blocks compared with the baseline (fixation) blocks in the various tasks. Cells tested in each Stroop task were classified as follows: (1) high-conflict specific, responding to the high- but not low-conflict word blocks; (2) graded, responding to both highand low-conflict blocks, but with a greater response to the highconflict blocks; (3) nonspecific, responding similarly to high- and low-conflict blocks or only to low-conflict blocks; or (4) not responsive, no statistically significant response to high- or lowconflict blocks. Of the 32 cells tested in the Stroop tasks, 14 (44\%) responded either exclusively to one of the high-conflict tasks or with a greater response to the high- than low-conflict tasks (Table $1)$. These conflict responses included both excitatory and inhibitory type responses (Tables 1-3). Also of note were seven cells responsive in the ES but not the CS task.

\section{Discussion}

The implication of the cACC in cognitive processes such as attention, salience (novelty and behavioral relevance), interference, and response competition has been determined by neuroimaging (Bush et al., 2000; Casey et al., 2000; Braver et al., 2001; Ruff et al., 2001; Downar et al., 2002; Botvinick et al., 2004) and lesion (Fellows and Farah, 2005) data. We now provide electrophysiological data at the single-cell level for an involvement of the cACC in attention-demanding tasks, including those with an emotional overlay.

The data indicate that cognitively demanding tasks can alter the firing rate of neurons in the human cACC. The novel finding is that one group of cells responds preferentially or predominantly to high-conflict versus low-conflict tasks. This finding may explain the relatively low incidence of task responses in our previous study of low-demand tasks (Davis et al., 2000). Impor- 
Table 1. Individual cell responses and classification

\begin{tabular}{|c|c|c|c|c|c|c|c|c|c|c|c|c|}
\hline \multirow[b]{2}{*}{ Patient } & \multirow[b]{2}{*}{ Age/sex } & \multirow[b]{2}{*}{ Side } & \multirow[b]{2}{*}{ Cell number } & \multicolumn{3}{|c|}{ Cell classification } & \multicolumn{6}{|c|}{ Task response } \\
\hline & & & & $\mathrm{CS}$ & $E S(G)$ & $\overline{E S}(0)$ & Arith & CS-low & CS-high & ES-low & ES-high & $O C D$ \\
\hline \multirow[t]{4}{*}{1} & $40 / \mathrm{M}$ & R & 1 & & GR+ & NS- & & & & - & + & - \\
\hline & & $\mathrm{R}$ & 2 & & & & 0 & & & & & \\
\hline & & $\mathrm{R}$ & 3 & $\mathrm{nr}$ & $\mathrm{nr}$ & $\mathrm{nr}$ & & 0 & 0 & 0 & 0 & 0 \\
\hline & & $\mathrm{R}$ & 4 & $\mathrm{nr}$ & $\mathrm{nr}$ & HS- & & 0 & 0 & 0 & 0 & - \\
\hline \multirow[t]{4}{*}{2} & $66 / F$ & R & 5 & NS + & NS + & & & + & + & + & 0 & \\
\hline & & R & 6 & $\mathrm{nr}$ & GR+ & & & 0 & 0 & + & + & \\
\hline & & R & 7 & & & & - & & & & & \\
\hline & & R & 8 & & & & + & & & & & \\
\hline \multirow[t]{7}{*}{3} & $26 / F$ & $\mathrm{R}$ & 9 & & & & + & & & & & \\
\hline & & R & 10 & & & & 0 & & & & & \\
\hline & & R & 11 & & $\mathrm{HS}+$ & $\mathrm{nr}$ & & & & 0 & + & 0 \\
\hline & & R & 12 & & $\mathrm{nr}$ & HS- & & & & 0 & 0 & - \\
\hline & & R & 13 & & $\mathrm{nr}$ & HS- & & & & 0 & 0 & - \\
\hline & & R & 14 & & $\mathrm{HS}+$ & $\mathrm{HS}+$ & - & & & 0 & + & + \\
\hline & & R & 15 & & & & - & & & & & \\
\hline \multirow[t]{3}{*}{4} & $27 / M$ & R & 16 & & & & + & & & & & \\
\hline & & R & 17 & & & & + & & & & & \\
\hline & & R & 18 & & GR+ & $\mathrm{GR}+$ & & & & + & + & + \\
\hline \multirow[t]{12}{*}{5} & $54 / F$ & R & 19 & & & & + & & & & & \\
\hline & & R & 20 & & & & + & & & & & \\
\hline & & R & 21 & & & & + & & & & & \\
\hline & & R & 22 & & NS + & $\mathrm{nr}$ & & & & + & + & 0 \\
\hline & & R & 23 & & & & 0 & & & & & \\
\hline & & R & 24 & & & & + & & & & & \\
\hline & & R & 25 & & & & + & & & & & \\
\hline & & R & 26 & NS + & $\mathrm{nr}$ & $\mathrm{nr}$ & & + & + & 0 & 0 & 0 \\
\hline & & R & 27 & $\mathrm{nr}$ & NS- & NS- & & 0 & 0 & - & 0 & - \\
\hline & & L & 28 & & & & 0 & & & & & \\
\hline & & $\mathrm{L}$ & 29 & & & & - & & & & & \\
\hline & & L & 30 & $\mathrm{GR}+$ & $\mathrm{HS}+$ & $\mathrm{nr}$ & + & + & + & 0 & + & 0 \\
\hline \multirow[t]{3}{*}{6} & $58 / \mathrm{M}$ & $\mathrm{R}$ & 31 & & HS- & $\mathrm{nr}$ & + & & & 0 & - & 0 \\
\hline & & $\mathrm{R}$ & 32 & NS + & NS + & NS + & & + & + & + & + & + \\
\hline & & $\mathrm{R}$ & 33 & NS- & HS- & $\mathrm{HS}+$ & + & - & 0 & 0 & - & + \\
\hline \multirow[t]{6}{*}{7} & $41 / \mathrm{F}$ & $\mathrm{R}$ & 34 & $\mathrm{nr}$ & $\mathrm{HS}+$ & & 0 & 0 & 0 & 0 & + & \\
\hline & & R & 35 & NS- & $\mathrm{GR}+$ & & 0 & - & 0 & - & + & \\
\hline & & R & 36 & $\mathrm{nr}$ & NS- & GR- & & 0 & 0 & - & - & - \\
\hline & & R & 37 & $\mathrm{nr}$ & NS+ & $\mathrm{nr}$ & & 0 & 0 & + & 0 & 0 \\
\hline & & L & 38 & & & & 0 & & & & & \\
\hline & & L & 39 & & & & - & & & & & \\
\hline \multirow[t]{8}{*}{8} & $28 / \mathrm{F}$ & $\mathrm{R}$ & 40 & $\mathrm{nr}$ & & NS- & & 0 & 0 & - & & - \\
\hline & & $\mathrm{R}$ & 41 & & & NS + & & & & + & & 0 \\
\hline & & R & 42 & & & NS- & & & & - & & 0 \\
\hline & & R & 43 & & & $\mathrm{nr}$ & & & & 0 & & 0 \\
\hline & & R & 44 & NS + & & $\mathrm{nr}$ & & + & + & 0 & & 0 \\
\hline & & $R$ & 45 & $\mathrm{nr}$ & & $\mathrm{nr}$ & & 0 & 0 & 0 & & 0 \\
\hline & & $R$ & 46 & $\mathrm{nr}$ & & & & 0 & 0 & & & \\
\hline & & $R$ & 47 & & & $\mathrm{nr}$ & & & & - & & 0 \\
\hline \multirow[t]{3}{*}{9} & $46 / R$ & R & 48 & $\mathrm{HS}-$ & $\mathrm{nr}$ & & & 0 & - & 0 & 0 & \\
\hline & & R & 49 & $\mathrm{NS}+$ & $\mathrm{nr}$ & & 0 & + & 0 & 0 & 0 & \\
\hline & & L & 50 & NS- & NS- & NS- & & - & 0 & - & 0 & 0 \\
\hline
\end{tabular}

L, Left; R, right; HS, high-contrast specific; GR, graded; NS, nonspecific; nr, no response; CS, counting Stroop; ES (G), general emotional Stroop; ES (0), OCD emotional Stroop; Arith, mental arithmetic task; +, excitation response; - , inhibition response; 0 , no response; blank, not tested.

tantly, these data indicate that human cACC neurons can respond to task conflict, difficulty, or response interference effects. The data also highlight the importance of human recordings, given that studies of nonhuman primates indicate that the primate ACC may not encode conflict (Ito et al., 2003; Nakamura et al., 2005); however, consistent with human neuroimaging responses to abrupt sensory, novel, task-relevant, or painful stimuli (Downar et al., 2002, 2003), our individual neuronal responses to high-conflict words, including those with emotional valence or OCD-specific meaning, may represent a simple neuron "salience response" in the human cACC.

Our findings impact on the simple scheme of the ACC divided into rostral "emotion" and caudal "cognition" parts. We now report that the $\mathrm{ACC}$ contains some neurons that respond differentially (either with excitation or inhibition) to high-conflict words that are emotionally laden but not to high-contrast words without emotional valence. Interestingly, the rostrocaudal ACC scheme described by Bush et al. (2000) based on neuroimaging studies included "deactivations" in the cACC during emotional tasks and in the rostral ACC during cognitive tasks (Bush et al., 2000). Although the meaning of neuroimaging deactivations is a contentious subject, it may involve inhibition of neuronal activity. Thus, each region may not be functionally exclusive.

For obvious ethical reasons, it is not possible to record from single ACC neurons in healthy individuals; therefore, one must recognize some limitations of generalizing data obtained in a 


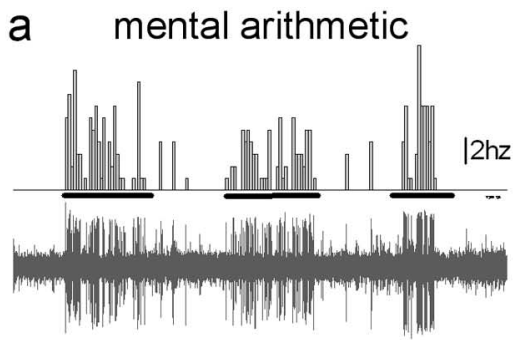

b non-specific excitation (cs)

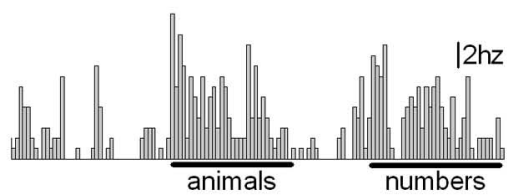

C non-specific inhibition (es)

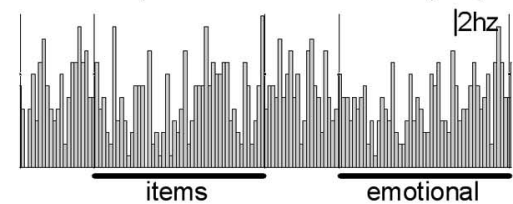

d

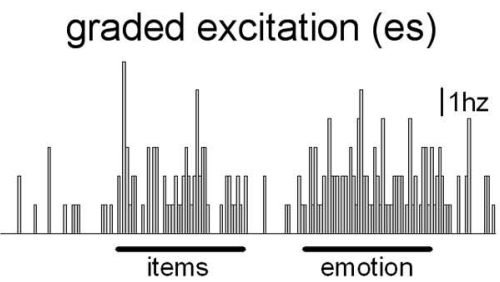

e

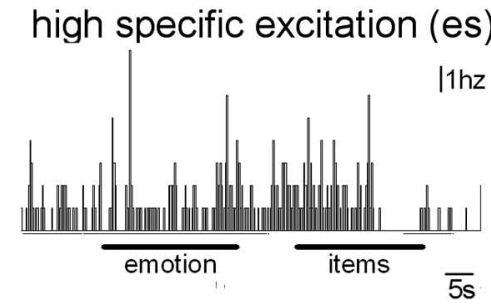

Figure 2. Example of cell response types. Electrophysiological recordings of a neuronal response during the mental arithmetic task $(\boldsymbol{a})$, a nonspecific excitatory response to the counting Stroop, $(\boldsymbol{b})$ and a nonspecific inhibitory response (c), graded response $(\boldsymbol{d})$, and high-conflict-specific response $(\boldsymbol{e})$ to the emotional Stroop are shown. Each panel shows the time histogram response of an individual neuron for tasks indicated by horizontal bars (1 s bin). $\boldsymbol{a}$, An example of a raw multiunit electrophysiological record is also shown.

Table 2. Incidence of responses ${ }^{a}$

\begin{tabular}{lrlrl}
\hline Task & Excitation & Inhibition & No response & Number tested \\
\hline Mental arithmetic & $12(48 \%)^{b}$ & $5(20 \%)$ & $8(32 \%)$ & 25 \\
CS, low conflict & $6(30 \%)$ & $3(15 \%)$ & $11(55 \%)$ & 20 \\
CS, high conflict & $5(25 \%)$ & $1(5 \%)$ & $14(70 \%)$ & 20 \\
ES, low conflict & $7(22 \%)$ & $8(26 \%)$ & $16(52 \%)$ & 31 \\
ES, general high conflict & $10(41 \%)$ & $3(13 \%)$ & $11(46 \%)$ & 24 \\
ES, OCD high conflict & $4(16 \%)$ & $7(28 \%)$ & $14(56 \%)$ & 25
\end{tabular}

${ }^{a}$ Response type based on the presence of a statistically significant change $(t$ test; $p<0.05)$ in firing rate during the conflict blocks compared with fixation blocks.

${ }^{b}$ Number of cells (\%).

Table 3. Cell types

\begin{tabular}{lllll}
\hline & High-conflict specific & Graded & Nonspecific & NR \\
\hline Counting Stroop $(n=20)$ & $1(\mathrm{I})$ & $1(\mathrm{E})$ & $8(5 \mathrm{E}, 3 \mathrm{ll})$ & 10 \\
Emotional Stroop $(\mathrm{G})(n=24)$ & $6(4 \mathrm{E}, 2 \mathrm{l})$ & $4(\mathrm{E})$ & $7(4 \mathrm{E}, 3 \mathrm{I})$ & 7 \\
Emotional Stroop $(0)(n=25)$ & $5(2 \mathrm{E}, 3 \mathrm{Il})$ & $2(1 \mathrm{E}, 1 \mathrm{ll})$ & $7(2 \mathrm{E}, 5 \mathrm{II})$ & 11 \\
\hline
\end{tabular}

Classification is based on a statistical comparison of the firing rate of each cell during the low-conflict and high-conflict blocks ( $t$ test; $p<0.5)$. $G, G$ General emotional Stroop; 0, OCD emotional Stroop; NR, no response; E, excitatory response; I, inhibitory response.

small number of patients with OCD to the healthy population. First, the reaction-time data indicate that these patients were somewhat slower ( $\sim 150-200 \mathrm{~ms})$ than healthy control subjects (Bush et al., 1998; Whalen et al., 1998; Seminowicz et al., 2004); however, this is likely because of a speed-accuracy tradeoff, because they rarely made any errors. Second, the emotional tasks likely induced stronger interference and cognitive effects in the OCD patients than they would in healthy individuals. Although these factors may have facilitated our identification of Stroopresponsive neurons and possibly even contributed to amplified neuronal sensitivities, our main findings of the existence of Stroop-responsive neurons in the ACC are likely to be generally applicable to a "normal" population. Another potential confound is that the number of neurons and tasks that were tested varied from patient to patient. These limitations were unavoidable because of the nature of the experimental conditions (i.e., awake patients undergoing a surgical procedure); however, of note is that taskresponsive neurons were identified in each patient. Finally, to avoid potential habituation effects that have been reported previously (Bush et al., 1998; Whalen et al., 1998), we minimized the number of Stroop test runs for each cell. Furthermore, many minutes typically elapsed between the identification and testing of different cells in any particular patient.

One implication from these data and previous functional MRI findings is that the effectiveness of surgical procedures for OCD may have its basis in the disruption of the activity of general cognitively responsive neurons. Although we did identify cACC neurons responsive in the ES but not the CS task, their numbers were relatively small. The ultimate impact of ES-specific responsive neurons on behavior and cognition may depend on the balance of activity contributed by this neuron type within the caudal and rostral ACC. Therefore, surgical procedures directed at altering emotional processes may need to target more rostral regions of the ACC, where (on the basis of functional neuroimaging) there are presumably more ESspecific excitatory neurons. Although not currently available, future surgical procedures within the rostral ACC should directly test this hypothesis.

\section{References}

Botvinick MM, Cohen JD, Carter CS (2004) Conflict monitoring and anterior cingulate cortex: an update. Trends Cogn Sci 8:539-546.

Braver TS, Barch DM, Gray JR, Molfese DL, Snyder A (2001) Anterior cingulate cortex and response conflict: effects of frequency, inhibition and errors. Cereb Cortex 11:825-836.

Bush G, Whalen PJ, Rosen BR, Jenike MA, McInerney SC, Rauch SL (1998) The counting Stroop: an interference task specialized for functional neuroimaging-validation study with functional MRI. Hum Brain Mapp 6:270-282.

Bush G, Luu P, Posner MI (2000) Cognitive and emotional influences in anterior cingulate cortex. Trends Cogn Sci 4:215-222.

Casey BJ, Thomas KM, Welsh TF, Badgaiyan RD, Eccard CH, Jennings JR, Crone EA (2000) Dissociation of response conflict, attentional selection, and expectancy with functional magnetic resonance imaging. Proc Natl Acad Sci USA 97:8728-8733.

Davis KD, Hutchison WD, Lozano AM, Tasker RR, Dostrovsky JO (2000) Human anterior cingulate cortex neurons modulated by attentiondemanding tasks. J Neurophysiol 83:3575-3577.

Downar J, Crawley AP, Mikulis DJ, Davis KD (2002) A cortical network 
sensitive to stimulus salience in a neutral behavioral context across multiple sensory modalities. J Neurophysiol 87:615-620.

Downar J, Mikulis DJ, Davis KD (2003) Neural correlates of the prolonged salience of painful stimulation. NeuroImage 20:1540-1551.

Fellows LK, Farah MJ (2005) Is anterior cingulate cortex necessary for cognitive control? Brain 128:788-796.

Hutchison WD, Davis KD, Lozano AM, Tasker RR, Dostrovsky JO (1999) Pain-related neurons in the human cingulate cortex. Nat Neurosci 2:403-405.

Ito S, Stuphorn V, Brown JW, Schall JD (2003) Performance monitoring by the anterior cingulate cortex during saccade countermanding. Science 302:120-122.

Mayberg HS, Lozano AM, Voon V, McNeely HE, Seminowicz D, Hamani C, Schwalb JM, Kennedy SH (2005) Deep brain stimulation for treatmentresistant depression. Neuron 45:651-660.

Nakamura K, Roesch MR, Olson CR (2005) Neuronal activity in macaque SEF and ACC during performance of tasks involving conflict. J Neurophysiol 93:884-908.

Pillay PK, Hassenbusch SJ (1992) Bilateral MRI-guided stereotactic cingulotomy for intractable pain. Stereotact Funct Neurosurg 59:33-38.

Richter EO, Davis KD, Hamani C, Hutchison WD, Dostrovsky JO, Lozano AM (2004) Cingulotomy for psychiatric disease: microelectrode guidance, a callosal reference system for documenting lesion location, and clinical results. Neurosurgery 54:622-628.

Ruff CC, Woodward TS, Laurens KR, Liddle PF (2001) The role of the anterior cingulate cortex in conflict processing: evidence from reverse Stroop interference. NeuroImage 14:1150-1158.

Seminowicz DA, Mikulis DJ, Davis KD (2004) Cognitive modulation of pain-related brain responses depends on behavioral strategy. Pain 112:48-58.

Whalen PJ, Bush G, McNally RJ, Wilhelm S, McInerney SC, Jenike MA, Rauch SL (1998) The emotional counting Stroop paradigm: a functional magnetic resonance imaging probe of the anterior cingulate affective division. Biol Psychiatry 44:1219-1228.

Williams ZM, Bush G, Rauch SL, Cosgrove GR, Eskandar EN (2004) Human anterior cingulate neurons and the integration of monetary reward with motor responses. Nat Neurosci 7:1370-1375. 